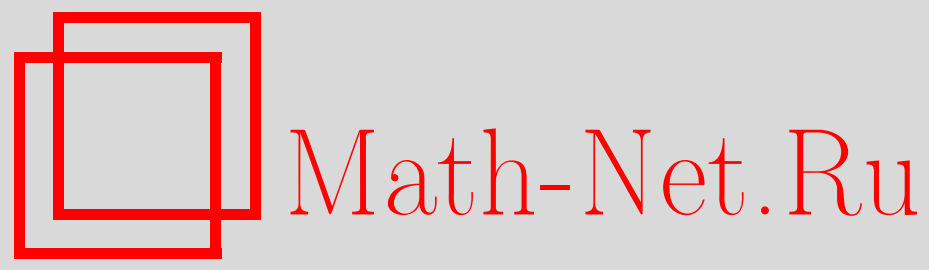

А. Г. Костюченко, А. А. Шкаликов, М. Ю. Юркин, Об устойчивости волчка с полостью, заполненной вязкой жидкостью, Функи. анализ и его прил., 1998, том 32, выпуск 2, 36-55

DOI: https://doi.org/10.4213/faa350

Использование Общероссийского математического портала MathNet.Ru подразумевает, что вы прочитали и согласны с пользовательским соглашением http://www . mathnet.ru/rus/agreement

Параметры загрузки:

IP: 54.198 .64 .247

26 апреля 2023 г., $15: 25: 41$

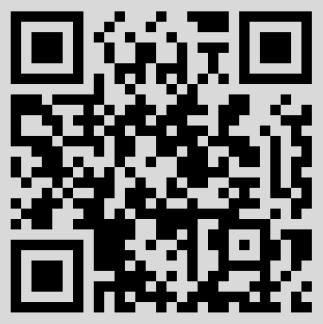


Функииональньй анализ и еәо приложения

1998, т. 32, вып. 2, с. 36-55

УДК 517.9

\section{Об устойчивости волчка с полостью, заполненной вязкой жидкостью}

(c) 1998. А. Г. КостЮченко, А. А. ШкАликов, М. Ю. ЮРКин

\section{Введение}

В работе изучаются малые колебания вращающегося волчка с полостью, целиком заполненной несжимаемой вязкой жидкостью. Распределение масс в теле и форма полости считаются произвольными. В невозмушенном стационарном состоянии жидкость имеет распределение скоростей твердого тела, вращающегося вместе с волчком вокруг вертикальной оси, являющейся главной осью инерции системы. Оболочка имеет неподвижную точку. Движение происходит в поле силы тяжести. Центр тяжести системы находится на главной оси инерции системы, вокруг которой совершалось невозмущенное движение.

Основная цель работы - получить критерий устойчивости движения такой системы и исследовать спектральные свойства оператора эволюции, соответствуюшего линеаризованным уравнениям движения. Эта задача имеет давнюю историю. Отметим в первую очередь исследования Соболева [1], Румянцева [2] и Черноусько [3], в которых даются достаточные условия устойчивости и признаки неустойчивости волчка (см. также монографии $[4,5])$. Румянцев $[2,4]$ показал, что волчок устойчив, если положительны оба числа

$$
n_{1}=a_{0}-a_{1}-k / \omega^{2} \quad \text { и } \quad n_{2}=a_{0}-a_{2}-k / \omega^{2} .
$$

Здесь $\omega$ - угловая скорость вращения волчка, а смысл других постоянных будет указан в $\S 1$. В частности, если волчок имеет ось симметрии выше второго порядка, то $a_{1}=a_{2}$ и для устойчивости достаточно положительности одного числа. В отсутствие силы тяжести этот достаточный признак устойчивости сводится к тому, чтобы ось невозмущенного вращения волчка была осью наибольшего центрального момента инерции. Румянцев получил этот признак способом, основанным на применении второго метода Ляпунова, а ранее Соболев получил его для идеальной жидкости (т.е. при нулевой вязкости), показав, что оператор эволюции соответствующей линейной задачи самосопряжен в гильбертовом пространстве с индефинитной метрикой, причем метрика дефинитна, если $n_{1}=n_{2}>0$. С другой стороны, Черноусько [3]

^ Исследования А. Г. Костюченко выполнены при поддержке РФФИ, гранты № 96-1596091 и № 96-01-00333, А. А. Шкаликова - при поддержке РФФИ, грант № 98-01-01000, и программы Volkswagen Stiftung (RIP program in Oberwolfach), М. Ю. Юркина - при поддержке РФФИ, грант № 96-15-96091, и ISSEP, грант а97-1559. 
получил признаки неустойчивости волчка в рассматриваемой задаче. Он нашел асимптотические разложения решений линеаризованных уравнений движения волчка в ряды по степеням числа Рейнольдса и показал, что в отсутствие силы тяжести при большой вязкости жидкости волчок будет неустойчив, если момент инерции системы относительно оси невозмущенного вращения является наименьшим или средним. У него были некоторые предположения о симметрии полости, которые затем были сняты Смирновой [22]. Она также показала, что вращение вокруг оси наибольшего момента инерции системы будет единственным устойчивым вращением, если жидкость имеет достаточно малую вязкость, а полость имеет форму тора [6]. Для волчка со сферической полостью этот результат получен Черноусько [7]. В неявной форме признаки неустойчивости волчка получил также Румянцев [4].

В настоящей работе мы показываем, что линеаризованное уравнение рассматриваемой задачи можно записать в такой форме, что соответствующий оператор эволюции является диссипативным в пространстве с индефинитной метрикой (в пространстве Понтрягина). Это дает возможность применить нетривиальные результаты теории таких операторов и полностью решить задачу о необходимых и достаточных условиях устойчивости и, более того, точно указать индекс неустойчивости. Предположения о симметрии волчка, отсутствии силы тяжести, большой или малой вязкости оказываются излишними. Кроме того, в отличие от предыдущих работ мы исследуем устойчивость системы (существенно бесконечномерной) в целом, а не только ее конечномерной «твердотельной» части. Один из основных результатов настоящей работы состоит в том, что индекс неустойчивости задачи не зависит ни от вязкости (если она ненулевая), ни от формы волчка и полости, и равен числу отрицательных собственных значений матрицы $\left(\begin{array}{cc}n_{1} & 0 \\ 0 & n_{2}\end{array}\right)$. При
нулевой вязкости это не так $($ см. $[1,8])$.

В методологическом отношении наш подход примыкает к работе Соболева [1]. Однако уравнения движения системы, рассмотренные Соболевым, были записаны им в системе координат, равномерно вращающейся в пространстве с угловой скоростью невозмущенного вращения волчка. Они оказываются сложными для анализа; их представление в операторной форме возможно только при нулевой вязкости (см. §5). Следуя [4], мы пользуемся системой координат, жестко связанной с телом. Полученные нами линеаризованные уравнения (cр. $[8,9])$ допускают удобную операторную трактовку.

Работа состоит из пяти параграфов. В $\S 1$ мы уточняем постановку задачи и выписываем уравнения движения системы. В 22 линеаризованные уравнения задачи представлены в виде операторного уравнения в гильбертовом пространстве. Показано, что оператор $\mathbf{T}$ эволюции системы является максимально диссипативным в пространстве Понтрягина. В $\S 3$ находится число собственных значений оператора $\mathbf{T}$ в нижней полуплоскости и доказывается, что это число совпадает с индексом неустойчивости. В $\$ 4$ исследуются базисные свойства собственных функций оператора $\mathbf{T}$ и дается описание его спектра при больших значениях вязкости. В 55 рассматривается случай симметричного волчка. Приводится семейство инвариантных подпространств оператора эволюции т и выделены те подпространства, на которых волчок 
может быть неустойчив. Здесь же указана связь изучаемого нами оператора T с оператором Соболева.

\section{§1. Уравнения движения волчка с жидкостью}

Обозначения. Пространства и операторы гидромеханики. Пусть твердое тело занимает ограниченный объем $\Omega_{1}$ в пространстве $\mathbb{R}^{3}$ и содержит внутри полость $\Omega$, целиком заполненную жидкостью, т.е. $\Omega \subset \Omega_{1}$. Далее считаем, что $\Omega$ есть область в $\mathbb{R}^{3}$. Само тело без жидкости, занимающее объем $\Omega_{1} \backslash \Omega$, назовем оболочкой. Мы будем оперировать понятием тела с затвердевшей жидкостью, подразумевая следующее: весь объем $\Omega_{1}$ рассматривается как твердое тело, плотность которого в $\Omega_{1} \backslash \Omega$ совпадает с прежней, а в области $\Omega$ - с плотностью жидкости. Тело движется вокруг неподвижной точки, которую мы примем за начало координат. Пусть $e_{0}, e_{1}, e_{2}$ - единичные векторы, выходящие из точки $O$ и совпадающие по направлениям с главными осями инерции тела с затвердевшей жидкостью. Рассмотрим ортонормированные реперы $O e_{0} e_{1} e_{2}$ и $O f_{0} f_{1} f_{2}$, первый из которых жестко связан с телом, а второй фиксирован в пространстве. В невозмущенном состоянии вектор $e_{0}$ совпадает с $f_{0}$. Состояние рассматриваемой системы (тело+жидкость) описывается тремя величинами $(z, w, v(x))$, где $z$ - координаты вектора $f_{0}, w$ - угловая скорость оболочки, а $v(x)$ поле скоростей жидкости. Здесь $x=\left(x_{0}, x_{1}, x_{2}\right) \in \Omega$ - координаты точки, причем все четыре величины $z, w, v(x)$ и $x$ рассматриваются как векторы в репере $O e_{0} e_{1} e_{2}$, жестко связанном с телом.

Из предположения о конечности кинетической энергии жидкости следует, что значения $v$ поля скоростей жидкости лежат в подпространстве $J_{0}(\Omega) \subset$ $L_{2}^{3}(\Omega)$. Подпространство $J_{0}(\Omega)$ получается замыканием в $L_{2}^{3}$ всех бесконечно дифференцируемых и финитных в $\Omega$ соленоидальных векторных полей (т. е. вектор-функций, для которых $\operatorname{div} v=0)$. Известно разложение Вейля

$$
L_{2}^{3}(\Omega)=J_{0}(\Omega) \oplus G(\Omega) .
$$

Здесь $G(\Omega)$ - подпространство функций вида $\operatorname{grad} p(x)$, где $p(x)$ - скалярная локально квадратично суммируемая функция, первые обобщенные частные производные которой принадлежат $L_{2}(\Omega)$.

Определяемые далее операторы хорошо известны в гидромеханике (см. [10] и [5]). Вектор

$$
B^{*} v=\int_{\Omega}[x, v(x)] d x
$$

где $[\cdot, \cdot]$ - векторное произведение в $\mathbb{R}^{3}$, называется гиростатическим моментом жидкости. Очевидно, что $B^{*}: J_{0}(\Omega) \rightarrow \mathbb{R}^{3}$ есть ограниченный оператор, а сопряженный к нему определяется равенством $B w=P_{0}([w, x])$, где $P_{0}$ - оператор ортогонального проектирования пространства $L_{2}^{3}(\Omega)$ на подпространство $J_{0}(\Omega)$. Легко проверить, что $\operatorname{rot} B w \equiv 2 w$. Известно [4], что это тождество в случае односвязной области может служить определением оператора $B$, т. е. оператор $B: \mathbb{R}^{3} \rightarrow J_{0}(\Omega)$, удовлетворяющий этому тождеству, однозначно определен. 
Пусть $\Delta$ - оператор Лапласа на подпространстве $J_{0}(\Omega)$, область определения которого составляют бесконечно дифференцируемые финитные в $\Omega$ функции $v \in J_{0}(\Omega)$. Оператор $R=P_{0} \Delta$, очевидно, неотрицателен, а потому определено его расширение по Фридрихсу, которое будем по-прежнему обозначать через $R$. Построенный оператор называется оператором Cтокса. Информацию об области определения оператора $R$ в случае негладкой области получить трудно, но область определения порождаемой им квадратичной формы (или область определения корня $R^{1 / 2}$ ) находится легко: она совпадает с линеалом $J_{0}(\Omega) \cap \stackrel{\circ}{H}_{1}(\Omega)$, где $\stackrel{\circ}{H}_{1}(\Omega)$ - пространство, получающееся замыканием множества гладких финитных в $\Omega$ функций в метрике пространства Соболева $H_{1}(\Omega)$. Известно [10], что оператор Стокса равномерно положителен и имеет дискретный спектр.

Вместо вещественного пространства $\mathbb{R}^{3}$ далее рассматривается его комплексификация $\mathbb{C}^{3}$. В пространстве $\mathbb{C}^{3}$ рассмотрим операторы $A$ и $A_{0}$, определяемые квадратичными формами

$$
(A w, w)=\int_{\Omega_{1}}\|[w, x]\|^{2} d \rho(x), \quad\left(A_{0} w, w\right)=\int_{\Omega_{1} \backslash \Omega}\|[w, x]\|^{2} d \rho(x),
$$

где $\rho(x)$ - распределение масс в оболочке $\Omega_{1} \backslash \Omega$ и жидкости в $\Omega$. Матрицы, отвечающие этим операторам, определяют тензоры инерции относительно точки $O$ тела с затвердевшей жидкостью и оболочки соответственно. Согласно определению репера $O e_{0} e_{1} e_{2}$, имеем $A=\operatorname{diag}\left\{a_{0}, a_{1}, a_{2}\right\}$, где $a_{j}$ - моменты инерции тела с затвердевшей жидкостью относительно главных осей инерции $O x_{j}$. Далее предполагается, что $A_{0}>0$. С физической точки зрения это предположение означает, что оболочка не является невесомой.

$\mathrm{B}$ пространстве $\mathbb{C}^{3}$ рассмотрим также оператор $H$, определяемый равенством

$$
i H x=\left[x, e_{0}\right] \quad \text { или } \quad H=\left(\begin{array}{ccc}
0 & 0 & 0 \\
0 & 0 & -i \\
0 & i & 0
\end{array}\right) \text {. }
$$

Оператор

$$
G=-2 P_{0} H,
$$

действующий в пространстве $J_{0}(\Omega)$, называется гироскопическим. Очевидно, что $G$ является ограниченным и самосопряженным. Через $I$ обозначается тождественный оператор как в пространстве $\mathbb{C}^{3}$, так и в пространстве $J_{0}(\Omega)$.

Далее в уравнениях фигурируют константы $\nu$ и $k$. Через $\nu$ обозначается вязкость жидкости (предполагается, что $\nu \neq 0$, если не оговорено противное). Через $k$ обозначается величина опрокидывающего момента силы тяжести, отнесенная к косинусу угла между векторами $f_{0}$ и $e_{0}$. Справедливо равенство $k=g l m$, где $l$ - расстояние от центра тяжести системы до неподвижной точки, $m$ - масса системы, а $g$ - ускорение силы тяжести. Плотность жидкости без ограничения общности далее предполагается равной единице. 
Уравнения движения волчка с жидкостью. Эволюция системы описывается уравнениями (см., например, [4])

$$
\begin{aligned}
\dot{z} & =[z, w], \\
A \dot{w}+B^{*} \dot{v} & =\left[A w+B^{*} v, w\right]+k\left[z, e_{0}\right], \\
\nabla p+\nu \Delta v & =\dot{v}+(v \cdot \nabla) v+2[w, v]+[w,[w, x]]+[\dot{w}, x], \quad \operatorname{div} v=0 .
\end{aligned}
$$

Первое из этих уравнений есть кинематическое соотношение, причем вектор $z$ называется вектором Пуассона. Второе уравнение выражает закон об изменении кинетического момента системы относительно точки $O$. В третей строке записано уравнение Навье-Стокса движения жидкости в подвижной системе координат, связанной с твердым телом. Применив к этому уравнению оператор проектирования $P_{0}$ и заметив, что

$$
P_{0} \nabla p=0, \quad P_{0}[w,[w, x]]=0, \quad P_{0}(u \cdot \nabla) u=P_{0}([v, \operatorname{rot} v]),
$$

получим уравнение вида

$$
B \dot{w}+\dot{v}=P_{0}([v,(2 w+\operatorname{rot} v)])+\nu P_{0} \Delta v .
$$

Линеаризуем первые два уравнения эволюции системы и полученное третье уравнение относительно стационарного решения системы (3) вида

$$
z_{0}=e_{0}, \quad w_{0}=\omega e_{0}, \quad v_{0}=0,
$$

где $\omega-$ постоянная (угловая скорость невозмущенного движения).

Подставим в систему (1) вместо функций $z, w, v$ их сдвиги $z_{0}+z, w_{0}+w$, $v_{0}+v$ и оставим только линейные члены. После простых выкладок с учетом (2) получим линеаризованные уравнения движения

$$
\begin{aligned}
\dot{z} & =i \omega\left(H z-\omega^{-1} H w\right), \\
A \dot{w}+B^{*} \dot{v} & =i \omega\left(k \omega^{-1} H z-H\left(a_{0} I-A\right) w+H B^{*} v\right), \\
B \dot{w}+\dot{v} & =i \omega\left(-2 P_{0} H+i \nu \omega^{-1} P_{0} \Delta\right) v .
\end{aligned}
$$

Эти уравнения удобнее изучать в операторной форме.

\section{$\S 2$. Операторное уравнение, соответствующее линеаризованным уравнениям эволюции системы, и его анализ}

Линеаризованные уравнения в операторной форме. Рассмотрим гильбертово пространство

$$
\mathfrak{H}=\mathbb{C}^{3} \times \mathbb{C}^{3} \times J_{0}(\Omega),
$$

элементами которого являются векторы $\mathbf{u}=(z, w, v)^{t}$. Здесь индекс $t$ означает транспонирование, т. е. элементы пространства $\mathfrak{H}$ суть столбцы, первые две компоненты которых лежат в $\mathbb{C}^{3}$, а последняя - в $J_{0}(\Omega)$. Операторы в пространстве $\mathfrak{H}$ естественно записывать в виде $(3 \times 3)$-матриц, компоненты которых сами являются операторами, действующими в пространствах $\mathbb{C}^{3}$, 
$J_{0}(\Omega)$ и между ними. Очевидно, систему (4) можно переписать в операторной форме

$$
\mathbf{W} \dot{\mathbf{u}}=i \omega \mathbf{M u}, \quad \mathbf{u} \in \mathfrak{H},
$$

где операторы $\mathbf{W}$ и $\mathbf{M}$ определены оператор-матрицами

$$
\mathbf{W}=\left(\begin{array}{ccc}
I & 0 & 0 \\
0 & A & B^{*} \\
0 & B & I
\end{array}\right), \quad \mathbf{M}=\left(\begin{array}{ccc}
H & -\omega^{-1} H & 0 \\
k \omega^{-1} H & H\left(A-a_{0} I\right) & H B^{*} \\
0 & 0 & D
\end{array}\right) .
$$

Здесь оператор $D$ определен в пространстве $J_{0}(\Omega)$ равенством

$$
D=G+i \frac{\nu}{\omega} R
$$

где $G$ - гироскопический оператор, а $R$ - оператор Стокса. Все фигурирующие здесь операторы, кроме одного - оператора Стокса $R$, - ограничены. В качестве области определения оператора $\mathbf{M}$ берем область

$$
\mathscr{D}(\mathbf{M})=\mathbb{C}^{3} \times \mathbb{C}^{3} \times \mathscr{D}(R) .
$$

Анализ уравнения (5) затруднен тем, что оператор $\mathbf{M}$ не обладает какимилибо специальными свойствами; он не является ни симметрическим, ни диссипативным. Наша идея состоит в том, чтобы «угадать» оператор $\mathbf{S}$, такой, что уравнение (5) после применения к нему оператора $\mathbf{S}$ примет более симметричную форму, а именно, оператор SM станет диссипативным, а оператор SW останется самосопряженным. Положим

$$
\mathbf{S}=\left(\begin{array}{ccc}
\omega^{2}(A+N) & -\omega I & 0 \\
-\omega A & I & 0 \\
-\omega B & 0 & I
\end{array}\right), \quad \text { где } N=\left(\begin{array}{ccc}
1 & 0 & 0 \\
0 & n_{1} & 0 \\
0 & 0 & n_{2}
\end{array}\right)
$$

а числа $n_{1}$ и $n_{2}$ определены в (1).

ПРЕДЛОЖЕНИЕ 1. Справедливы представления

$$
\begin{gathered}
\mathbf{J}:=\mathbf{S W}=\left(\begin{array}{ccc}
\omega^{2}(A+N) & -\omega A & -\omega B^{*} \\
-\omega A & A & B^{*} \\
-\omega B & B & I
\end{array}\right), \\
\mathbf{L}:=\mathbf{S M}=\left(\begin{array}{ccc}
\left(\omega^{2} a_{0}-2 k\right) H & -\omega H A+k \omega^{-1} H & -\omega H B^{*} \\
-\omega A H+k \omega^{-1} H & A H+H A-a_{0} H & H B^{*} \\
-\omega B H & B H & D
\end{array}\right) .
\end{gathered}
$$

ДокАЗАТЕЛЬСтво. В справедливости выписанных равенств убеждаемся непосредственной проверкой с учетом равенства $N H=\left(a_{0} I-A-k \omega^{-2} I\right) H$.

ПРЕДЛОЖЕНИЕ 2. Оператор $\mathbf{S}$ ограничен и обратим в пространстве $\mathfrak{H}$, если невырожденна матрица $N$.

ДокАЗАТЕЛЬСтво. Имеем

$$
\left(\begin{array}{cc}
\omega^{2}(A+N) & -\omega I \\
-\omega A & I
\end{array}\right)=\left(\begin{array}{cc}
I & -\omega I \\
0 & I
\end{array}\right)\left(\begin{array}{cc}
\omega^{2} N & 0 \\
0 & I
\end{array}\right)\left(\begin{array}{cc}
I & 0 \\
-\omega A & I
\end{array}\right)
$$


откуда вытекает обратимость оператора $\mathbf{S}$ при условии, что $n_{1} n_{2} \neq 0$.

ПРЕДЛОЖЕНИЕ 3. Оператор W равномерно положителен в пространcmве $\mathfrak{H}$.

ДокАЗАтЕльство. Заметим, что оператор $\mathbf{W}$ есть конечномерное возмущение единичного оператора в $\mathfrak{H}$. Поэтому в силу альтернативы Фредгольма достаточно показать, что $\mathbf{W}>0$. Из определений операторов $A, A_{0}$ и $B$ получаем

$$
(\mathbf{W u}, \mathbf{u})=(z, z)+\left(A_{0} w, w\right)+\int_{\Omega}([w, x]+v(x),[w, x]+v(x)) d x .
$$

Следовательно, $\mathbf{W} \geqslant 0$. Если $\mathbf{W u}=0$, то $z=0$ и $w=0$ (в силу ранее сделанного предположения $\left.A_{0}>0\right)$. Но тогда имеем также $v(x) \equiv 0$.

ПРЕДЛОЖЕНИЕ 4. Оператор Ј является самосопряженным, ограниченньм и обратимым в пространстве $\mathfrak{H}$, если матрииа $N$ невырожденна. Еао отрицательный спектр состоит из конечного числа собственньх значений, причем число отричательных собственных значений оператора $\mathbf{J} u$ матриць $N$ совпадают.

ДокАЗАтЕльство. В пространстве $\mathfrak{H}$ рассмотрим операторы, определяемые матрицами

$$
\mathbf{J}_{0}=\left(\begin{array}{ccc}
\omega^{2} N & 0 & 0 \\
0 & A-B^{*} B & 0 \\
0 & 0 & I
\end{array}\right), \quad \mathbf{S}_{0}=\left(\begin{array}{ccc}
I & 0 & 0 \\
-\omega I & I & 0 \\
-\omega B & B & I
\end{array}\right) .
$$

В силу предложения 3 оператор $\mathbf{W}$ равномерно положителен, а потому этим же свойством обладает оператор

$$
\left(\begin{array}{cc}
A & B^{*} \\
B & I
\end{array}\right)=\left(\begin{array}{cc}
I & 0 \\
B & I
\end{array}\right)\left(\begin{array}{cc}
A-B^{*} B & 0 \\
0 & I
\end{array}\right)\left(\begin{array}{cc}
I & B^{*} \\
0 & I
\end{array}\right)
$$

Следовательно, $A-B^{*} B \gg 0$ и $\pi_{-}\left(\mathbf{J}_{0}\right)=\pi_{-}(N)$, где через $\pi_{-}$обозначается число отрицательных собственных значений операторов. Далее, простая проверка показывает справедливость равенства $\mathbf{J}=\mathbf{S}_{0}^{*} \mathbf{J}_{0} \mathbf{S}_{0}$, откуда вытекает, что $\mathbf{J}$ обратим; кроме того, $\pi_{-}(\mathbf{J})=\pi_{-}\left(\mathbf{J}_{0}\right)=\pi_{-}(N)$. Предложение доказано.

Напомним, что оператор $\mathbf{C}$ называется диссипативным, если $\operatorname{Im}(\mathbf{C x}, \mathbf{x}) \geqslant 0$ для всех $\mathbf{x} \in \mathscr{D}(\mathbf{C})$, и максимально диссипативным, если нижняя открытая полуплоскость $\mathbb{C}^{-}$(или хотя бы одна точка $\mu \in \mathbb{C}^{-}$) принадлежит резольвентному множеству оператора $\mathbf{C}$. Далее мы будем иметь дело с диссипативными операторами в пространстве Понтрягина. Пусть $\mathbf{J}-$ самосопряженный ограниченный и обратимый оператор, причем $\pi_{-}(\mathbf{J})=\varkappa<\infty$. Гильбертово пространство $\mathfrak{H}$ с индефинитным скалярным произведением $(\mathbf{J} \mathbf{x}, \mathbf{x})$ называется пространством Понтрягина и обозначается $\Pi_{\varkappa}=\{\mathbf{J}, \mathfrak{H}\}$. Оператор $\mathbf{C}$ называется диссипативным в пространстве $\Pi_{\varkappa}$, если $\operatorname{Im}(\mathbf{J C x}, \mathbf{x}) \geqslant 0$ для всех $\mathbf{x} \in \mathscr{D}(\mathbf{C})$, и максимально диссипативным, если найдется точка $\mu \in \mathbb{C}^{-}$(но не обязательно вся полуплоскость $\mathbb{C}^{-}$), принадлежащая резольвентному множеству оператора С. Известно (см. [11, гл. 2, теорема 2.10]), 
что $\mathbf{C}$ максимально диссипативен в $\Pi_{\varkappa}$ в том и только том случае, когда JC максимально диссипативен в $\mathfrak{H}$.

Основной смысл преобразований, проведенных выше, заключался в подготовке доказательства следующего результата.

Теорема 1. В случае невырожденности матрицы $N$ уравнение (5) эволючии системь эквивалентно уравнению

$$
\dot{\mathbf{u}}=i \omega \mathbf{T u},
$$

в котором оператор $\mathbf{T}=\mathbf{J}^{-1} \mathbf{L}$ с областью определения $\mathbb{C}^{3} \times \mathbb{C}^{3} \times \mathscr{D}(R)$ является максимально диссипативньм в пространстве Понтрягина $\Pi_{\varkappa}=$ $\{\mathbf{J}, \mathfrak{H}\}$, əдe $\varkappa=\pi_{-}(N)$.

ДоказАтельство. Имеем $\operatorname{Im}(\mathbf{J T} \mathbf{x}, \mathbf{x})=\operatorname{Im}(\mathbf{L x}, \mathbf{x}) \geqslant 0$ для всех $\mathbf{x} \in \mathscr{D}(\mathbf{T})$. Это неравенство вытекает из представления

$$
\mathbf{L}=\mathbf{V}+\mathbf{D}, \quad \mathbf{D}=\operatorname{diag}\{I, I, D\}, \quad D=G+i \nu \omega^{-1} R,
$$

где $\mathbf{V}$ - самосопряженный конечномерный оператор, $G=G^{*}$ - гироскопический оператор, а $R=R^{*} \gg 0$ - оператор Стокса.

Очевидно, что оператор $\mathbf{D}$ является максимально диссипативным в пространстве $\mathfrak{H}$. Поскольку $\mathbf{V}$ самосопряжен и ограничен, этим же свойством обладает и $\mathbf{L}$. Но тогда оператор $\mathbf{T}$ является максимально $\mathbf{J}$-диссипативным, так как он не имеет нетривиальных $\mathbf{J}$-диссипативных расширений. Отметим, что максимальная диссипативность оператора $\mathbf{T}$ в пространстве $\Pi_{\varkappa}$ вытекает также из дискретности его спектра, что доказано ниже.

ЗАмЕчАниЕ 1. Полезно отметить следующий факт. Если

$$
\mathbf{S}_{1}=\left(\begin{array}{ccc}
\left(\omega^{2} N\right)^{-1} & A(\omega N)^{-1} & (\omega N)^{-1} B^{*} \\
(\omega N)^{-1} & (A+N) N^{-1} & N^{-1} B^{*} \\
0 & 0 & I
\end{array}\right)
$$

TO

$$
\begin{aligned}
\mathbf{J}_{1}:=\mathbf{W S}_{1}= & \left(\begin{array}{ccc}
\left(\omega^{2} N\right)^{-1} & A(\omega N)^{-1} & (\omega N)^{-1} B^{*} \\
A(\omega N)^{-1} & A(A+N) N^{-1} & (A+N) N^{-1} B^{*} \\
B(\omega N)^{-1} & B(A+N) N^{-1} & B N^{-1} B^{*}+I
\end{array}\right), \\
\mathbf{L}_{1} & :=\mathbf{M S}_{1}=\left(\begin{array}{ccc}
0 & -\omega^{-1} H & 0 \\
-\omega^{-1} H & -a_{0} H & 0 \\
0 & 0 & D
\end{array}\right) .
\end{aligned}
$$

При этом оператор $\mathbf{S}_{1}$ обратим, что следует из невырожденности матрицы $\left(\begin{array}{cc}\omega^{-2} & A \omega^{-1} \\ \omega^{-1} & A+N\end{array}\right)$. Таким образом, уравнение (5) после замены $\mathbf{u}=\mathbf{S}_{1} \mathbf{f}$ приводится к виду

$$
\mathbf{J}_{1} \dot{\mathbf{f}}=i \omega \mathbf{L}_{1} \mathbf{f}, \quad \text { или } \quad \dot{\mathbf{f}}=i \omega \mathbf{T}_{1} \mathbf{f}, \quad \mathbf{T}_{1}=\mathbf{J}_{1}^{-1} \mathbf{L}_{1},
$$

где $\mathbf{J}_{1}$ - самосопряженный, а $\mathbf{L}_{1}$ - максимально диссипативный операторы в $\mathfrak{H}$. При этом операторы $\mathbf{J}_{0}, \mathbf{J}, \mathbf{J}_{1}$ конгруэнтны, а именно

$$
\mathbf{J}_{1}=\mathbf{S}_{1}^{*} \mathbf{J} \mathbf{S}_{1}, \quad \text { где } \mathbf{J}=\mathbf{S}_{0}^{*} \mathbf{J}_{0} \mathbf{S}_{0} .
$$


Следовательно, оператор $\mathbf{J}_{1}$ порождает метрику Понтрягина, причем $\pi_{-}\left(\mathbf{J}_{1}\right)$ $=\pi_{-}(\mathbf{J})=\pi_{-}\left(\mathbf{J}_{0}\right)=\pi_{-}(N)$. Указанная замена приводит оператор $\mathbf{M} \mathrm{K}$ наиболее удобному виду: прямой сумме операторов в пространствах $\mathbb{C}^{3} \times \mathbb{C}^{3}$ и $J_{0}(\Omega)$, что используется в дальнейшем. Заметим, что операторы $\mathbf{L}$ и $\mathbf{L}_{1}$ также конгруэнтны: $\mathbf{L}_{1}=\mathbf{S}_{1}^{*} \mathbf{L} \mathbf{S}_{1}$.

\section{§3. Критерий устойчивости и индекс неустойчивости}

Необходимым условием устойчивости уравнения (6) (т.е. ограниченности всех его решений) является отсутствие собственных значений в открытой нижней полуплоскости у оператора $\mathbf{T}$. Следующий результат дает описание спектра оператора $\mathbf{T}$ в замкнутой нижней полуплоскости.

Tеорема 2. Cпектр оператора T дискретен. Единственной точкой его спектра на вещественной оси является точка $\lambda=0$, которой отвечают два собственньх вектора $\mathbf{x}_{0}=\left(e_{0}, 0,0\right)^{t}, \mathbf{x}_{1}=\left(0, e_{0}, 0\right)^{t}$. Подпространство Ker $\mathbf{T}$ является $\mathbf{J}$-положительным, присоединенных векторов, отвечающих нулевому собственному значению, нет. В открытой нижней полуплоскости имеется ровно $\varkappa=\pi_{-}(N)$ собственных значений оператора $\mathbf{T}$. $B$ частности, при $\varkappa=0$ всл полуплоскость $\mathbb{C}^{-}$принадлежит резольвентному множеству оператора $\mathbf{T}$.

ДокаЗАТЕЛЬСтво. Положим $\mathbf{D}=\operatorname{diag}(I, I, D)$. Очевидно, что оператор $\mathbf{D}^{-1}$ компактен в $\mathfrak{H}$ (это следует из компактности оператора $R^{-1}$ в пространстве $\left.J_{0}(\Omega)\right)$. Имеем

$$
\mathbf{T}-\lambda \mathbf{I}=\mathbf{J}^{-1}\left[\mathbf{I}+(\mathbf{V}-\lambda \mathbf{J}) \mathbf{D}^{-1}\right] \mathbf{D}, \quad \mathbf{V}=\mathbf{L}-\mathbf{D},
$$

где $\mathbf{V}$ - конечномерный оператор. Так как оператор $\mathbf{T}$ максимально диссипативный, то его резольвентное множество непусто. Тогда из выписанного представления и теоремы о голоморфной оператор-функции (см. [12, гл. 1]), следует дискретность спектра оператора $\mathbf{T}$.

Покажем, что $\mathbf{T}$ не имеет ненулевых вещественных собственных значений. Так как спектр $\mathbf{T}$ дискретен, то достаточно показать, что $\operatorname{Ker}(\mu \mathbf{J}-\mathbf{L})=0$ при $0 \neq \mu \in \mathbb{R}$. Пусть

$$
(\mu \mathbf{J}-\mathbf{L}) \mathbf{u}=0, \quad \mathbf{u}=(z, w, v)^{t} \in \mathfrak{H}, \quad 0 \neq \mu \in \mathbb{R} .
$$

Тогда $0=\operatorname{Im}((\mu \mathbf{J}-\mathbf{L}) \mathbf{u}, \mathbf{u})=(R v, v)$; поэтому $v=0$. Пользуясь невырожденностью оператора $\mathbf{S}$, из равенства $\mathbf{S}(\mu \mathbf{W}-\mathbf{M})=\mu \mathbf{J}-\mathbf{L}$ находим $(\mu \mathbf{W}-\mathbf{M})\{z, w, 0\}$. Из определения операторов $\mathbf{W}$ и $\mathbf{M}$ теперь последовательно получаем

$$
B w=0, \quad w=0, \quad H z=0, \quad \text { и } \quad(\mu I-H) z=0,
$$

что влечет за собой $\mathbf{u}=0$. Таким образом, оператор $\mathbf{T}$ не имеет ненулевых вещественных собственных значений.

Покажем, что нуль является собственным значением, и вычислим собственный вектор. Пусть $\mathbf{T u}=0$. Из равенства $\mathbf{T}=\mathbf{W}^{-1} \mathbf{M}$ имеем $\mathbf{M u}=0$. Пользуясь матричным представлением оператора $\mathbf{M}$, находим, что его ядро 
состоит из двух линейно независимых векторов $\mathbf{x}_{0}=\left(e_{0}, 0,0\right)^{t}$ и $\mathbf{x}_{1}=$ $\left(0, e_{0}, 0\right)^{t}$. Положим $\mathbf{x}=\alpha \mathbf{x}_{0}+\beta \mathbf{x}_{1}$. Тогда

$$
(\mathbf{J} \mathbf{x}, \mathbf{x})=\omega^{-2}|\alpha|^{2}+a_{0}\left(a_{0}+1\right)|\beta|^{2}+2 a_{0} \omega^{-1} \operatorname{Re}(\alpha \bar{\beta})>0,
$$

если $\mathbf{x} \neq 0$, т.е. ядро оператора $\mathbf{T}$ является $\mathbf{J}$-положительным подпространством. В этом случае присоединенных векторов к собственным векторам $\mathbf{x} \in \operatorname{Ker} \mathbf{T}$ быть не может (см. [11, гл. 2]).

Для доказательства утверждения о числе собственных значений оператора T в нижней полуплоскости воспользуемся фундаментальной теоремой о существовании инвариантных подпространств для максимальных диссипативных операторов в пространстве Понтрягина. По существу эта теорема была доказана Понтрягиным [13] (хотя сформулирована в [13] только для самосопряженных операторов). В приведенной ниже (и даже более общей) формулировке она получена в работах Крейна и Лангера [14] и Азизова [15].

ТЕОРЕМА ОБ ИНВАРИАНТНЫХ ПОдПРОсТРАнСТВАХ. Пусть $\mathbf{T}-$ максимально диссипативный оператор в пространстве Понтрягина $\Pi_{\varkappa}=\{\mathbf{J}, \mathfrak{H}\}$. Тогда существует $\mathbf{T}$-инвариантное $\mathbf{J}$-неположительное подпространство

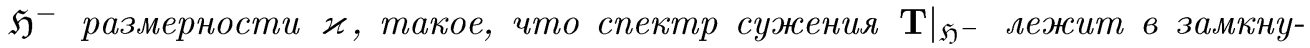
той нижней полуплоскости, а в открытой нижней полуплоскости $\mathbb{C}^{-}$он совпадает со спектром оператора $\mathbf{T}$.

Мы доказали, что вся вещественная ось, кроме нуля, принадлежит резольвентному множеству оператора $\mathbf{T}$. Нуль является собственным значением этого оператора, но соответствующее этому собственному значению подпространство является Ј-положительным. Следовательно, никакой ненулевой вектор этого подпространства не принадлежит Ј-неположительному инвариантному относительно $\mathbf{T}$ подпространству $\mathfrak{H}^{-}$. Поэтому спектр сужения $\left.\mathbf{T}\right|_{\mathfrak{H}^{-}}$лежит в открытой полуплоскости $\mathbb{C}^{-}$. Но, согласно сформулированной выше теореме, в $\mathbb{C}^{-}$он совпадает со спектром оператора $\mathbf{T}$; следовательно, число собственных значений оператора $\mathbf{T}$ в $\mathbb{C}^{-}$совпадает с числом $\varkappa=\operatorname{dim} \mathfrak{H}^{-}=\pi_{-}(N)$.

Назовем индексом неустойчивости уравнения (6) размерность факторпространства линейного пространства всех решений по отношению к пространству его ограниченных решений (т.е. число линейно независимых растущих решений по модулю ограниченных решений). Обозначим через $\nu(\mathbf{T})$ индекс неустойчивости уравнения (6). Очевидно, что $\nu(\mathbf{T})$ не меньше числа собственных значений оператора $\mathbf{T}$, лежащих в $\mathbb{C}^{-}$. Поэтому, согласно теореме $2, \nu(\mathbf{T}) \geqslant \pi_{-}(N)$. Конечно, в общей ситуации индекс неустойчивости может быть строго больше числа собственных значений соответствующего оператора в полуплоскости $\mathbb{C}^{-}$. Такие примеры известны (см., в частности, [16]). Однако в нашем случае имеет место равенство.

ТЕорема 3. B случае $n_{1} n_{2} \neq 0$ индекс неустойчивости $\nu(\mathbf{T})$ уравнения (6) совпадает с числом $\pi_{-}(N)$. В частности, задача устойчива тогда и только тогда, когда оба числа $n_{1} u n_{2}$ положительны.

ДокАЗАтЕльство. Если $N>0$, то оператор $\mathbf{J}$ равномерно положителен. Тогда метрика $(\mathbf{J x}, \mathbf{x})$ в пространстве $\mathfrak{H}$ эквивалентна исходной. В этой 
метрике оператор $T$ максимально диссипативный, а потому является генератором сжимающей полугруппы (см. [17, гл. 9]). Следовательно, всякое решение уравнения (6) (понимаемое как равенство в $\mathfrak{H}$ ) удовлетворяет условию $\left\|\mathbf{J}^{1 / 2} u(t)\right\| \leqslant\left\|\mathbf{J}^{1 / 2} u(0)\right\|$ при $t \geqslant 0$, т. е. задача устойчива.

Пусть $\pi_{-}(N)>0$. В этом случае задача неустойчива в силу неравенства $\nu(\mathbf{T}) \geqslant \pi_{-}(N)$. Вычислим индекс. Пусть $\mathfrak{H}_{-}-$(одномерное или двумерное) подпространство, образованное собственными векторами, отвечающими собственным значениям из полуплоскости $\mathbb{C}^{-}$. Покажем, что $\mathfrak{H}_{-}$есть Ј-отрицательное подпространство.

Пусть $\mathbf{x} \in \mathfrak{H}_{-}$, а $\mathbf{u}(t)$ — элементарное решение уравнения $(6)$ с начальным условием $\mathbf{u}(0)=\mathbf{x}$ (в случае $\pi_{-}(N)=1$ это решение имеет вид $\mathbf{u}(t)=$ $e^{i \omega \lambda_{0} t} \mathbf{x}$, где $\left.\lambda_{0} \in \mathbb{C}^{-}\right)$. Согласно (6), имеем

$$
\begin{aligned}
\frac{d}{d t}(\mathbf{J} \mathbf{u}, \mathbf{u}) & =(\mathbf{J} \dot{\mathbf{u}}, \mathbf{u})+(\mathbf{J} \mathbf{u}, \dot{\mathbf{u}})=(i \omega \mathbf{J T} \mathbf{u}, \mathbf{u})+(\mathbf{u}, i \omega \mathbf{J T} \mathbf{T}) \\
& =-2 \omega \operatorname{Im}(\mathbf{J T} \mathbf{T}, \mathbf{u})=-2 \nu(R v, v) .
\end{aligned}
$$

Очевидно, что $\mathbf{u}(t) \rightarrow 0$ при $t \rightarrow-\infty$. Интегрируя последнее равенство от $-\infty$ до 0, получим

$$
(\mathbf{J} \mathbf{x}, \mathbf{x})=-2 \nu \int_{-\infty}^{0}(R v(t), v(t)) d t
$$

Так как $R>0$, то $(\mathbf{J x}, \mathbf{x}) \leqslant 0$. Предположим, что $(\mathbf{J x}, \mathbf{x})=0$. Тогда $v(t) \equiv 0$. Выписав покоординатно уравнения $(5)$, легко получить, что $\dot{w}(t) \equiv 0$ и $\dot{z}(t) \equiv 0$. Следовательно, вектор $\mathbf{x}=(z, w, 0)^{t}$ принадлежит ядру оператора $\mathbf{T}$, т. е. отвечает нулевому собственному значению. Это влечет за собой $\mathbf{x}=0$.

Рассмотрим теперь оператор $\mathbf{J}^{-1} \mathbf{T}^{*} \mathbf{J}=\mathbf{J}^{-1} \mathbf{L}^{*}$. Из матричного представления оператора $\mathbf{L}$ вытекает, что $\mathscr{D}\left(\mathbf{J}^{-1} \mathbf{T}^{*} \mathbf{J}\right)=\mathscr{D}(\mathbf{T})$. Очевидно также, что оператор $-\mathbf{J}^{-1} \mathbf{T}^{*} \mathbf{J}$ максимально $\mathbf{J}$-диссипативный. Пусть $\mathfrak{H}_{-}^{*}$ - инвариантное подпространство оператора $\mathbf{J}^{-1} \mathbf{T}^{*} \mathbf{J}$, образованное собственными векторами, отвечающими собственным значениям из верхней полуплоскости. Повторив приведенные выше рассуждения, получим, что $\mathfrak{H}_{-}^{*}$ является J-отрицательным. Так как подпространство $\mathfrak{H}_{-}^{*}$ конечномерно, то оно равномерно J-отрицательно. В силу теоремы Понтрягина ([13, теорема 1]) подпространство $\mathfrak{H}_{+}$, являющееся J-ортогональным дополнением к $\mathfrak{H}_{-}^{*}$, равномерно J-положительно. Очевидно, что подпространство $\mathfrak{H}_{+}$является инвариантным относительно $\mathbf{T}$, а спектр сужения $\mathbf{T}_{+}=\left.\mathbf{T}\right|_{\mathfrak{H}_{+}}$лежит в замкнутой верхней полуплоскости. Таким образом, пространство $\mathfrak{H}$ разлагается в прямую сумму инвариантных относительно $\mathbf{T}$ равномерно $\mathbf{J}$-дефинитных подпространств $\mathfrak{H}_{-}$и $\mathfrak{H}_{+} \cdot$ Тогда уравнение $(6)$, рассмотренное на подпространстве $\mathfrak{H}_{+}$, очевидно, устойчиво. Поэтому индекс неустойчивости $\nu(\mathbf{T})$ равен $\operatorname{dim} \mathfrak{H}_{-}=\pi_{-}(N)$.

В дополнение к теореме 3 заметим, что неустойчивость уравнения (6) в целом влечет за собой неустойчивость движения волчка, т. е. твердой части системы. А именно, справедлив следующий результат. 
ТЕОрема 4. Пусть $\mathbf{u}(t)=(z(t), w(t), v(t))^{t}$ - неограниченное решение уравнения (6). Тогда Функиия $z(t)$ также неограничена.

ДокАЗАТЕльство. В ходе доказательства теоремы 3 найдено разложение пространства $\mathfrak{H}$ в прямую сумму инвариантных относительно $\mathbf{T}$ равномерно J-дефинитных подпространств: $\mathfrak{H}=\mathfrak{H}_{+}+\mathfrak{H}_{-}$. В частности, всякое растущее решение $\mathbf{u}(t)$ уравнения (6) представимо в виде

$$
\mathbf{u}(t)=\mathbf{u}_{+}(t)+\mathbf{u}_{-}(t), \quad \text { где }\left(\mathbf{J} \mathbf{u}_{-}(t), \mathbf{u}_{-}(t)\right) \rightarrow-\infty \text { при } t \rightarrow+\infty .
$$

Положим

$$
\mathbf{u}_{-}(t)=(z(t), w(t), v(t))^{t}, \quad \mathbf{u}_{-}^{0}(t):=\mathbf{S}_{0} \mathbf{u}_{-}(t)=\left(z(t), w_{0}(t), v_{0}(t)\right)^{t} .
$$

Тогда, согласно (7),

$$
\begin{aligned}
\left(\mathbf{J u}_{-}(t), \mathbf{u}_{-}(t)\right) & =\left(\mathbf{J}_{0} \mathbf{u}_{-}^{0}(t), \mathbf{u}_{-}^{0}(t)\right) \\
& =\omega^{2}(N z(t), z(t))+\left(\left(A-B^{*} B\right) w_{0}(t), w_{0}(t)\right)+\left(v_{0}(t), v_{0}(t)\right) .
\end{aligned}
$$

Так как два последних слагаемых в правой части положительны, а сумма стремится к $-\infty$ при $t \rightarrow+\infty$, то $z(t)$ - неограниченная функция.

ЗАмЕчАНИЕ 2. Оценка сверху числа собственных значений с отрицательной мнимой частью для оператора $\mathbf{T}_{1}=\mathbf{J}_{1}^{-1} \mathbf{L}_{1}$ получена в работе [9]. Утверждения теорем 2 и 3 о числе собственных значений в полуплоскости $\mathbb{C}^{-}$и индексе неустойчивости можно получить как следствие результатов из [18, 19$]$. Но здесь мы предпочли дать независимое доказательство.

\section{§4. Дополнительные свойства оператора T эволюции системы. Спектр при большой вязкости}

Числовой образ оператора Т и свойства собственных функций. Числовым образом оператора $F$ называют множество значений квадратичной формы $(F x, x)$, когда $x$ пробегает $\mathscr{D}(F),\|x\|=1$. Ясно, что спектр оператора лежит в числовом образе. Далее приведем понятие базиса для метода суммирования Абеля порядка $\alpha$, принадлежащее Лидскому [20]. Пусть оператор $F$ имеет дискретный спектр, причем все его собственные значения, за исключением конечного числа, лежат в угле $|\arg \lambda-\pi / 2|<\theta<\pi$. Для краткости считаем, что все собственные значения простые. Говорим, что система $\left\{y_{k}\right\}$ собственных функций оператора $F$, отвечающих собственным значениям $\left\{\lambda_{k}\right\}$, образует базис для метода суммирования Абеля порядка $\alpha$, $\alpha<2 \theta / \pi$, если для всех $f \in \mathfrak{H}$ ряд

$$
f(t)=\sum e^{\left(-i \lambda_{k}\right)^{\alpha} t}\left(f, z_{k}\right) y_{k}
$$

сходится при всех $t>0$ по норме пространства $\mathfrak{H}$ (после, возможно, заключения некоторых членов ряда в скобки, расстановка которых не зависит от $f$ и $t$ ), причем существует сильный предел ряда $f(t)$ при $t \rightarrow 0$, равный $f$. Здесь $\left\{z_{k}\right\}$ - система собственных функций сопряженного оператора, биортогональная к $\left\{y_{k}\right\}$, а $\left(-i \lambda_{k}\right)^{\alpha}$ понимается как значение главной ветви 
аналитической функции $\lambda^{\alpha}$. Очевидно, всякий базис для метода суммирования Абеля является полной системой в $\mathfrak{H}$.

Как и в предыдущем параграфе, мы обозначаем через $\mathbf{T}_{ \pm}$сужения генератора $\mathbf{T}$ уравнения (6) на инвариантные подпространства $\mathfrak{H}_{ \pm}$, найденные в теореме 2 .

ТЕОРема 5. Числовой образ оператора $\mathbf{T}_{+}$лежит в полуполосе $|\operatorname{Re} \lambda|<$ $c_{0}, \operatorname{Im} \lambda>-c_{1}$ nри некоторых постоянных $c_{0}, c_{1}>0$. Собственные значения $\lambda_{k}=\lambda_{k}(\mathbf{T})$ имеют асимптотику

$$
\lambda_{k}=i\left(\frac{3 \pi^{2} k}{\operatorname{mes}(\Omega)}\right)^{2 / 3} \frac{\nu}{\omega}(1+o(1)), \quad k=1,2,3, \ldots,
$$

и лежат в полуполосе указанного вида. Они симметричны относительно мнимой оси. Собственные векторы оператора $\mathbf{T}$ образуют базис для метода суммирования Абеля любого порядка $\alpha>1 / 2$. В частности, при $\alpha=1$ получаем, что любое решение уравнения (6) представляется при $t>0$ в виде сходящегося ряда по собственным функииям оператора Т. Оператор $i \mathbf{T}$ является генератором голоморфной полугруппь в пространстве $\mathfrak{H}$.

ДокАЗАТЕльство. Пусть $\mathbf{Q}_{+}$- ортопроектор на подпространство $\mathfrak{H}_{+}$

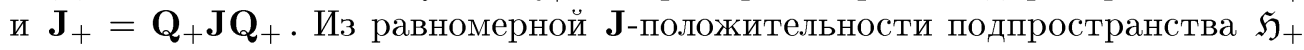
следует, что $\mathbf{J}_{+}-$равномерно положительный оператор в $\mathfrak{H}_{+} \cdot$ При $\mathbf{x} \in \mathfrak{H}_{+}$ имеем

$$
\begin{aligned}
(\mathbf{T x}, \mathbf{x}) & =\left(\mathbf{J}_{+} \mathbf{T} \mathbf{x}, \mathbf{J}_{+}^{-1} \mathbf{x}\right)=\left(\mathbf{J T} \mathbf{x}, \mathbf{J}_{+}^{-1} \mathbf{x}\right) \\
& =\left(\mathbf{L x}, \mathbf{J}_{+}^{-1} \mathbf{x}\right)=\left(\mathbf{Q}_{+} \mathbf{L} \mathbf{Q}_{+} \mathbf{x}, \mathbf{J}_{+}^{-1} \mathbf{x}\right) .
\end{aligned}
$$

Заметим, что если $C$ - равномерно положительный оператор, то вещественная форма $(F x, x)$ ограничена (полуограничена) тогда и только тогда, когда таковой является форма $(F x, C x)$. Из матричного представления диссипативного оператора $\mathbf{L}$ вытекает, что вешественная часть формы $(\mathbf{L x}, \mathbf{x})$ ограничена, а ее мнимая часть полуограничена. Следовательно, тем же свойством обладает форма (9). Поэтому числовой образ оператора $\mathbf{T}_{+}$лежит в полуполосе. Вне числового образа $W\left(\mathbf{T}_{+}\right)$известна оценка резольвенты

$$
\left\|\left(\lambda-\mathbf{T}_{+}\right)^{-1}\right\| \leqslant 1 / d\left(\lambda, W\left(\mathbf{T}_{+}\right)\right),
$$

где $d-$ расстояние от $\lambda$ до $W\left(\mathbf{T}_{+}\right)$. В частности, резольвента имеет максимальное убывание $\left(\leqslant c|\lambda|^{-1}\right)$, если $\lambda$ асимптотически лежит вне малого угла, содержащего мнимую ось. Из известных результатов теории полугрупп (см. $\left[17\right.$, гл. 9]) получаем, что $i \mathbf{T}_{+}$есть генератор голоморфной полугруппы в пространстве $\mathfrak{H}_{+}$. Поскольку $\mathbf{T}=\mathbf{T}_{+}+\mathbf{T}_{-}$, где $\mathbf{T}_{-}$конечномерен, то этим же свойством обладает оператор $i \mathbf{T}$.

Положим $\mathbf{R}=\operatorname{diag}(I, I, R)$, где $R$ - оператор Стокса. Имеем

$$
\mathbf{J}=\mathbf{I}+\mathbf{K}, \quad \mathbf{L}=\left(i \nu \omega^{-1} \mathbf{I}+\mathbf{V}\right) \mathbf{R}, \quad \mathbf{V}=\left(\mathbf{L}-i \nu \omega^{-1} \mathbf{R}\right) \mathbf{R}^{-1},
$$

где $\mathbf{K}$ - конечномерный, a $\mathbf{V}$ - компактный операторы. Поэтому $\mathbf{T}=$ $i \nu \omega^{-1}\left(\mathbf{I}+\mathbf{V}_{1}\right) \mathbf{R}$, где $\mathbf{V}_{1}-$ компактный оператор. 
Собственные значения операторов $\mathbf{R}$ и $R$ совпадают, кроме одного, $\lambda=1$. Асимптотика собственных значений оператора Стокса $R$ известна [5] и имеет вид (8) (с точностью до коэффициента $\nu / \omega)$. Согласно теореме КелдышаГохберга-Крейна (см. [12, гл. 5]), аналогичная асимптотика сохраняется и для компактного возмущения $\mathbf{T}$ оператора $\mathbf{R}$. Симметричность собственных значений пучка $\lambda \mathbf{J}-\mathbf{L}$ или оператора $\mathbf{T}$ относительно мнимой оси вытекает из вещественности матриц $A, N, i H$.

Утверждение о суммируемости методом Абеля достаточно доказать для оператора $\mathbf{T}_{+}$в пространстве $\mathfrak{H}_{+}$. Вне полуполосы мы доказали оценку резольвенты (10). Очевидно, сингулярные числа операторов $\mathbf{T}^{-1}$ и $\mathbf{R}^{-1}$ имеют одинаковую асимптотику (см. $\left[12\right.$, гл. 2]), т.е. $s_{k}(\mathbf{T}) \sim k^{p}, p=2 / 3$. Но тогда утверждение о базисе для суммирования методом Абеля порядка $\alpha>$ $p^{-1}-1=1 / 2$ сразу следует из результата статьи [21].

Спектр оператора $\mathbf{T}$ при большой вязкости. С физической точки зрения естественно, что спектр оператора эволюции системы при вязкости $\nu \rightarrow \infty$ будет приближаться к спектру оператора задачи о движении волчка с затвердевшей жидкостью.

Уравнение малых колебаний волчка с затвердевшей жидкостью как целого твердого тела, вращающегося вокруг вертикальной оси, получается проекцией уравнения (5) на подпространство $\mathbb{C}^{3} \times \mathbb{C}^{3} \times\{0\}$ в пространстве $\mathfrak{H}$. Следовательно, спектр соответствующей задачи совпадает со спектром матричного пучка

$$
V(\lambda)=\lambda\left(\begin{array}{ll}
I & 0 \\
0 & A
\end{array}\right)-\left(\begin{array}{cc}
H & \omega^{-1} H \\
k \omega^{-1} H & H\left(A-a_{0} I\right)
\end{array}\right)=: \lambda W^{(s)}-M^{(s)},
$$

где $W^{(s)}$ и $M^{(s)}$ — проекции операторов $\mathbf{W}$ и $\mathbf{M}$ на $\mathbb{C}^{3} \times \mathbb{C}^{3}$ — «тердотельную» часть пространства $\mathfrak{H}$. Очевидно, что нуль является двукратным собственным значением пучка $V(\lambda)$. Рассмотрим операторы $\mathbf{S}_{1}, \mathbf{J}_{1}$, $\mathbf{L}_{1}$, определенные в замечании 1. Заметим, что собственные значения пучков $\mathbf{V}(\lambda)=\lambda \mathbf{W}-\mathbf{M}$ и $\mathbf{V}_{1}(\lambda)=\lambda \mathbf{J}_{1}-\mathbf{L}_{1}$ совпадают; то же самое справедливо и для их проекций на $\mathbb{C}^{3} \times \mathbb{C}^{3}$. А именно, матричные пучки $V(\lambda)$ и

$$
\begin{aligned}
V_{1}(\lambda)=V(\lambda) S_{1}^{(s)} & =\lambda\left(\begin{array}{cc}
\left(\omega^{2} N\right)^{-1} & A(\omega N)^{-1} \\
A(\omega N)^{-1} & A(A+N) N^{-1}
\end{array}\right)-\left(\begin{array}{cc}
0 & -\omega^{-1} H \\
-\omega^{-1} H & -a_{0} H
\end{array}\right) \\
& =: \lambda J_{1}^{(s)}-L_{1}^{(s)}
\end{aligned}
$$

имеют одинаковые спектры.

Теорема 6. Пусть $n_{1} n_{2} \neq 0$. При $\nu \rightarrow \infty$ все собственнье значения оператора $\mathbf{T}$ уходят на бесконечность, кроме шести собственньх значений, два из которых совпадают с нулем, а остальные четыре приближаются $\kappa$ ненулевыл собственныл значениям пучка $V(\lambda)$, соответствуючего твердотельной части системь. Скорость приближения оченивается величиной $O\left(\nu^{-1 / 2}\right)$, если все четыре - простые собственные значения, и величиной $O\left(\nu^{-1 / 4}\right)$, если они двукратны. Собственные значения матричных пучков $V(\lambda)$ и $V_{1}(\lambda)$ симметричнь относительно вещественной $и$ мнимой осей. Для собственньх значений, уходящих на $\infty$, справедливь формуль

$$
\lambda_{k}=\nu \omega^{-1} \lambda_{k}^{(f)}(1+o(1)) \quad \text { npu } \nu \rightarrow \infty,
$$


где $\lambda_{k}^{(f)}$ - ненулевые собственные значения пучка $\lambda\left(\begin{array}{cc}A & B^{*} \\ B & I\end{array}\right)-i\left(\begin{array}{cc}0 & 0 \\ 0 & R\end{array}\right)$
в пространстве $\mathbb{C}^{3} \times J_{0}(\Omega)$.

ДокАЗАТЕльство. Симметричность собственных значений пучка $V_{1}(\lambda)$ (а следовательно, и $V(\lambda)$ ) относительно вещественной оси следует из его самосопряженности, а относительно мнимой оси - из вещественности матриц $A, N, i H$.

Положим $\mu=\omega / \nu, \rho=\mu^{1 / 2}$ и умножим пучок $\lambda \mathbf{J}_{1}-\mathbf{L}_{1}$ слева и справа на оператор $\operatorname{diag}\left(I, I, \mu^{1 / 2} R^{-1 / 2}\right)$. Получим, что собственные значения оператора $\mathbf{T}$ совпадают с собственными значениями пучка

$$
\mathbf{S}_{\rho}(\lambda)=\lambda\left(\begin{array}{ll}
J_{1}^{(s)} & \rho C_{1}^{*} \\
\rho C_{1} & \rho^{2} C
\end{array}\right)-\left(\begin{array}{cc}
L_{1}^{(s)} & 0 \\
0 & \rho^{2} G+i I
\end{array}\right),
$$

где $C, C_{1}$ - некоторые ограниченные операторы, вид которых сейчас несуществен. Согласно теореме Реллиха-Като (см. [17, гл. 7]), при малых $\rho$ (или больших $\nu$ ) собственные значения пучка $\mathbf{S}_{\rho}(\lambda)$ лежат в малых окрестностях собственных значений пучка $\mathbf{S}_{0}(\lambda)$, т. е. в нуле, в окрестности $\infty$ и в окрестностях четырех ненулевых симметричных собственных значений пучка $V_{1}(\lambda)$.

В общем положении, когда эти ненулевые собственные значения простые, их возмущения аналитически зависят от $\rho$; поэтому скорость приближения пропорциональна величине $\rho=\mu^{1 / 2}$. Если же невозмущенные собственные значения двукратны, то для их возмущений справедливы разложения в ряды Пьюизо по степеням $\rho^{1 / 2}$; поэтому скорость приближения не превосходит величины $O\left(\mu^{1 / 4}\right)$. Собственных значений кратности, большей двух, нет в силу симметрии.

Заметим, что

$$
\rho(\lambda \mathbf{W}-\mathbf{M})=\rho \lambda \mathbf{W}-i \operatorname{diag}\{0,0, R\}+\mathbf{K}(\rho),
$$

где $\mathbf{K}(\rho)=O(\rho)$. Вновь применяя теорему Реллиха-Като, получим формулы (11) для собственных значений, уходящих на $\infty$.

Особый интерес представляют вещественные собственные значения пучка $V(\lambda)$, если они есть. Из какой полуплоскости и как приближаются к ним собственные значения оператора $\mathbf{T}$ при $\nu \rightarrow \infty$ ? Ответ дает следующая теорема.

Tеорема 7. Пусть $\lambda_{0}-$ простое вещественное собственное значение пучка $V(\lambda)$, a $h_{0}=\left(z_{0}, w_{0}\right)^{t}$ - соответствуюший собственный вектор. Тогда собственное значение $\lambda$ оператора $\mathbf{T}$, близкое $\kappa \lambda_{0}$, имеет вид

$$
\lambda=\lambda_{0}+c i \mu+O\left(\mu^{3 / 2}\right), \quad c=\frac{\omega \lambda_{0}^{2}\left(B^{*} R^{-1} B w_{0}, w_{0}\right)}{\left(J^{(s)} h_{0}, h_{0}\right)} .
$$

Здесь $J^{(s)}$ - сужение оператора $\mathbf{J} н а \mathbb{C}^{3} \times \mathbb{C}^{3}$. Таким образом, $\lambda$ находится в верхней или нижней полуплоскости в зависимости от знака иисла $\left(J^{(s)} h_{0}, h_{0}\right)$.

ДокаЗАТЕльство. Из представления (12) для пучка $\mathbf{S}_{\rho}(\lambda)$ и теоремы Реллиха-Като следует аналитическая зависимость собственного значения $\lambda$ оператора $\mathbf{T}$ и соответствующего собственного вектора $\mathbf{f}$ от параметра $\rho$, a 
именно

$$
\lambda=\lambda_{0}+c_{1} \rho+c_{2} \rho^{2}+\ldots, \quad \mathbf{f}=\mathbf{f}_{0}+\rho \mathbf{f}_{1}+\rho^{2} \mathbf{f}_{2}+\ldots
$$

Положим $\mathbf{f}_{j}=\left(g_{j}, r_{j}\right)^{t}, j=1,2,3$, где $g_{j}$ - проекции векторов $\mathbf{f}_{j}$ на $\mathbb{C}^{3} \times \mathbb{C}^{3}$. Выпишем первые коэффициенты разложения по степеням $\rho$ уравнения $\mathbf{S}_{\rho}(\lambda) \mathbf{f}=0$. Выписав первые два коэффициента, получим

$$
\begin{gathered}
\lambda_{0} J_{1}^{(s)} g_{0}-L_{1}^{(s)} g_{0}=0, \quad r_{0}=0, \\
\lambda_{0} C_{1} g_{0}-i r_{1}=0, \quad c_{1} J_{1}^{(s)} g_{0}+\lambda_{0} J_{1}^{(s)} g_{1}-L_{1}^{(s)} g_{1}-C_{1} r_{0}=0 .
\end{gathered}
$$

Из этих равенств имеем $c_{1}\left(J_{1}^{(s)} g_{0}, g_{0}\right)=0$. Известно, что собственный вектор самосопряженного пучка $V_{1}(\lambda)$, отвечающий простому вещественному собственному значению, является $J_{1}^{(s)}$-дефинитным. Поэтому $c_{1}=0$. Далее, коэффициент при $\rho^{2}$ равен

$$
c_{2} J_{1}^{(s)} g_{0}+\lambda_{0} C_{1}^{*} r_{1}+\lambda_{0} J_{1}^{(s)} g_{2}-L_{1}^{(s)} g_{2}=0 .
$$

Но тогда

$$
c_{2}\left(J_{1}^{(s)} g_{0}, g_{0}\right)-i \lambda_{0}^{2}\left(C_{1}^{*} C_{1} g_{0}, g_{0}\right)=0 .
$$

Собственный вектор $h_{0}=\left(z_{0}, w_{0}\right)^{t}$ пучка $V(\lambda)$, очевидно, равен $S_{1}^{(s)} g_{0}$. Выписав выражение для оператора $C_{1}$ в (12) с учетом равенств $\mathbf{W} \mathbf{S}_{1}=\mathbf{J}_{1}$ и $W^{(s)} S_{1}^{(s)}=J_{1}^{(s)}$, найдем, что

$$
C_{1} g_{0}=R^{-1 / 2} W^{(s)} h_{0}=R^{-1 / 2} B w_{0} .
$$

Из равенства $J_{1}^{(s)}=\left(S_{1}^{(s)}\right)^{*} J^{(s)} S_{1}^{(s)}($ cр. $(7))$ и равенства $(14)$ теперь находим выражение для коэффициента $c_{2}=i c$ в $(13)$.

ЗАмЕчание 3 . Знак числа $\left(J_{1}^{(s)} g_{0}, g_{0}\right)=\left(J^{(s)} h_{0}, h_{0}\right)$ определяет тип собственного значения $\lambda_{0}$ самосопряженного пучка $V_{1}(\lambda)$. В силу симметрии собственные значения $\lambda_{0}$ и $-\lambda_{0}$ имеют одинаковый тип. Если вещественных значений только два, то оба они положительного типа, так как в противном случае при больших $\nu$ в нижней полуплоскости будет находиться три собственных значения оператора $\mathbf{T}$, что невозможно. Если вещественных собственных значений четыре (что возможно лишь в случае $n_{1} n_{2}>0$ ), то их тип положителен, если оба числа $n_{1}$ и $n_{2}$ положительны, а в противном случае одна из пар симметричных собственных значений имеет отрицательный тип. В частности, утверждение теоремы 2 о числе собственных значений оператора $\mathbf{T}$ в нижней полуплоскости можно получить, опираясь только на теорему 7 и принимая во внимание, что ненулевых вещественных собственных значений нет.

ЗАмечАние 4 . Оператор $B^{*} R^{-1} B$ вычислен в [3] для некоторых форм полостей. Формула (13) получена в [3] и [22] в случае $k=0$, когда сила тяжести отсутствует.

Все рассуждения мы приводили в предположении, что $n_{1} n_{2} \neq 0$. Однако, поведение собственных значений оператора $\mathbf{T}$ можно исследовать без этого 
предположения. Интересно также проследить, как осуществляется переход собственного значения из верхней полуплоскости в нижнюю при изменении параметра угловой скорости $\omega$, когда $\omega$ проходит критическое значение.

ПреДЛОЖЕНИЕ 5. Пусть $\omega$ таково, ито $n_{1}=0$, а $n_{2} \neq 0$. Тогда нуль трехкратное полупростое собственное значение оператора $\mathbf{T}=\mathbf{T}(\omega)$. При мальх $\varepsilon$ у оператора $\mathbf{T}(\omega+\varepsilon)$ есть простое собственное значение $\lambda(\varepsilon)$, удовлетворяюшее равенству

$$
\lambda(\varepsilon)=\frac{2 k \varepsilon}{\omega^{2}\left(D^{-1} B e_{1}, B e_{1}\right)}+O\left(\varepsilon^{2}\right) \quad n p u \quad \varepsilon \rightarrow \pm 0 .
$$

Здесь $e_{1}$ - единичный вектор, определенный в 1 . Число $\left(D^{-1} B e_{1}, B e_{1}\right)$ чисто мнимое.

ДокАЗАТЕЛЬСТво. Проверка того, что нуль - трехкратное полупростое собственное значение, осуществляется так же, как в теореме 2 . В силу (7) собственные значения оператора $\mathbf{T}$ и пучка $\lambda \mathbf{J}_{0}-\mathbf{L}_{0}$, где $\mathbf{L}_{0}=\left(\mathbf{S}_{0}^{*}\right)^{-1} \mathbf{L} \mathbf{S}_{0}^{-1}$, совпадают. Для операторов $\mathbf{J}_{0}$ и $\mathbf{L}_{0}$ легко выписать их разложения по степеням $\varepsilon$, ограничившись первыми двумя членами. Несложно убедиться, что $\left(G B e_{1}, B e_{1}\right)=0$. Поэтому собственное значение $\lambda(\varepsilon)$ проходит через нуль по мнимой оси (здесь мы учитываем также симметрию). Далее доказательство проводится точно так же, как в теореме 7. Поэтому его детали мы опускаем.

\section{$\S 5$. Случай симметричного волчка}

Инвариантные подпространства. В случае симметрии можно выделить инвариантные подпространства оператора $\mathbf{T}$ и провести в них исследования на устойчивость. Пусть $O x_{0}$ - ось симметрии волчка порядка $p$. Иными словами, если волчок повернуть на угол $2 \pi / p$ вокруг оси $O x_{0}$, то он совместится сам с собою. Далее считаем, что $p>2$. Для тела вращения считаем, что $p=\infty$. Введем операторы

$$
U_{s}=\left(\begin{array}{ccc}
1 & 0 & 0 \\
0 & \cos 2 \pi s / p & \sin 2 \pi s / p \\
0 & -\sin 2 \pi s / p & \cos 2 \pi s / p
\end{array}\right), \quad \mathbf{U}_{s}=\left(\begin{array}{ccc}
U_{s} & 0 & 0 \\
0 & U_{s} & 0 \\
0 & 0 & V_{s}
\end{array}\right),
$$

где оператор $V_{s}$ определен равенством $V_{s} v=U_{s}\left(v\left(U_{-s} x\right)\right)$. В случае $p=\infty$ операторы $\mathbf{U}_{s}$ определим теми же равенствами, заменив $p$ на $2 \pi$.

ПРЕДЛОЖЕНИЕ 6. Если О ${ }_{0}$ - ось симметрии волчка порядка $p$, то при любом иелом $s$ (в случае $p=\infty$ при любом $s \in \mathbb{R}$ ) справедливы равенства

$$
A U_{s}=U_{s} A, \quad H U_{s}=U_{s} H, \quad B^{*} V_{s}=V_{s} B^{*}, \quad V_{s} D=D V_{s} .
$$

Операторьи $\mathbf{T}$ и $\mathbf{U}_{s}$ перестановочньл. Операторьь

$$
\mathbf{Q}_{j}=\frac{1}{p} \sum_{l=0}^{p-1} e^{2 \pi i l j / p} \mathbf{U}_{l} \quad\left(\mathbf{Q}_{j}=\frac{1}{2 \pi} \int_{0}^{2 \pi} e^{i j x} \mathbf{U}_{x} d x, \quad \text { если } p=\infty\right)
$$


являются ортопроекторами в $\mathfrak{H}$. При этом $\sum_{j=0}^{p-1} \mathbf{Q}_{j}=\mathbf{I}($ при $p=\infty$ $\left.\sum_{j=-\infty}^{\infty} \mathbf{Q}_{j}=\mathbf{I}\right)$. Подпространства $\mathfrak{H}_{j}=\mathbf{Q}_{j}(\mathfrak{H})$ являются инвариантныли относительно оператора $\mathbf{T}$.

ДоказАтельство. Равенство (15) следует из определения (см. лемму 1 из [8]). Остальные утверждения вытекают непосредственно из этих равенств. Отметим, что при $p=\infty$ интеграл в определении проекторов $\mathbf{Q}_{j}$ понимается в смысле Петтиса.

Легко видеть, что $\mathbf{Q}_{j}=\mathbf{Q}_{j-p}$. Вместо $\mathbf{Q}_{p-1}$ далее удобнее рассматривать $\mathbf{Q}_{-1}$.

ТЕОрема 8. В случае симметрии порлдка $p(2<p \leqslant \infty)$ уравнение (6) устойчиво в подпространствах $\mathfrak{H}_{j}$ при всех $j \neq \pm 1$. В подпространствах $\mathfrak{H}_{ \pm 1}$ уравнение имеет индекс неустойчивости 1 , если $n_{1}\left(=n_{2}\right)<0$.

ДокАЗАТЕЛЬСтво. Для определенности считаем $p<\infty$. Изменения при $p=\infty$ очевидны. Проекторы $\mathbf{Q}_{j}$ имеют вид $\operatorname{diag}\left(R_{j}, R_{j}, Q_{j}\right)$, причем

$$
\begin{gathered}
R_{-1}=\frac{1}{2}\left(\begin{array}{ccc}
0 & 0 & 0 \\
0 & 1 & i \\
0 & -i & 1
\end{array}\right), \quad R_{0}=\left(\begin{array}{ccc}
1 & 0 & 0 \\
0 & 0 & 0 \\
0 & 0 & 0
\end{array}\right), \\
R_{1}=\overline{R_{-1}}, \quad R_{j}=0 \text { при } j \neq 0, \pm 1,
\end{gathered}
$$

где $Q_{j}$ - ортопроектор в $J_{0}(\Omega)$ на векторные поля $v(x)$, которые после поворота на угол $2 \pi / p$ вокруг оси $O x_{0}$ совпадают с $e^{-2 \pi i j / p} v(x)$. Следовательно, при $j \neq 0, \pm 1$ уравнение $(6)$ в подпространстве $\mathfrak{H}_{j}=\{0\} \times\{0\} \times Q_{j}\left(J_{0}(\Omega)\right)$ имеет вид $\dot{v}=i \omega D v$ и в силу диссипативности оператора $D$ является устойчивым.

Подпространство $\mathfrak{H}_{0}$ имеет вид $\mathbb{C}_{0} \times \mathbb{C}_{0} \times Q_{0}\left(J_{0}(\Omega)\right)$. Из равенства $R_{0} B^{*}=B^{*} Q_{0}$ следует, что $\mathfrak{H}_{0}$ инвариантно относительно $\mathbf{L}$. Имеем $\mathbf{J}=$ $\mathbf{S}_{0}^{*} \mathbf{J}_{0} \mathbf{S}_{0}$. Заметим, что

$$
\mathbf{S}_{0}\left(\mathfrak{H}_{0}\right) \subset \mathbb{C} e_{0} \times \mathbb{C} e_{0} \times J_{0}(\Omega)=: \mathfrak{H}_{0}^{\prime},
$$

а на подпространстве $\mathfrak{H}_{0}^{\prime}$ оператор $\mathbf{J}_{0}$ положителен. Следовательно, $\mathbf{J}$ положителен на $\mathfrak{H}_{0}$. Но тогда оператор $\mathbf{T}=\mathbf{J}^{-1} \mathbf{L}$ является максимально диссипативным в скалярном произведении $(\mathbf{J} \mathbf{x}, \mathbf{x})$ на $\mathfrak{H}_{0}$, которое эквивалентно обычному, а потому уравнение (6) устойчиво на $\mathfrak{H}_{0}$.

Поскольку $\mathbf{T} \overline{\mathbf{u}}=-\overline{\mathbf{T u}}$ (здесь черта означает комплексное сопряжение), а $\mathfrak{H}_{-1}$ состоит из векторов, комплексно сопряженных к $\mathfrak{H}_{1}$, то индекс неустойчивости уравнения $(6)$ в $\mathfrak{H}_{1}$ и $\mathfrak{H}_{-1}$ одинаков. При $n_{1}<0$ индекс неустойчивости в $\mathfrak{H}_{1}$ не может быть нулем или большим единицы (иначе получаем противоречие с теоремой 3). Следовательно, он равен 1.

Связь с уравнениями Соболева. В случае симметричного волчка и идеальной жидкости $(\nu=0)$ Соболев [1] изучал оператор $\mathbf{F}$ в пространстве $\mathbb{C} \times \mathbb{C} \times J_{0}(\Omega)$, который определял следующим образом:

$$
\mathbf{F}(z, w, v)=\left(z^{1}, w^{1}, v^{1}\right) .
$$


Здесь числа $z^{1}, w^{1}$ и функция $v^{1}=\left(v_{0}^{1}, v_{1}^{1}, v_{2}^{1}\right) \in J_{0}(\Omega)$ находятся из уравнений

$$
\begin{gathered}
v_{0}^{1}=i \frac{\partial p}{\partial x_{0}}, \quad v_{2}^{1}=-2 \omega i v_{1}+i \frac{\partial p}{\partial x_{2}}+2 \omega w \frac{\partial \bar{\chi}}{\partial x_{1}} \\
v_{1}^{1}=2 \omega i v_{2}+i \frac{\partial p}{\partial x_{1}}-2 \omega w \frac{\partial \bar{\chi}}{\partial x_{2}}, \quad z^{1}=\omega w, \quad \operatorname{div} v=\operatorname{div} v^{1}=0, \\
\left.v_{n}^{1}\right|_{\partial \Omega}=0, \quad A_{1} w^{1}=A_{2} w+A_{3} z+\int_{\Omega}\left(v_{2} \frac{\partial \chi}{\partial x_{1}}-v_{1} \frac{\partial \chi}{\partial x_{2}}\right) d \Omega .
\end{gathered}
$$

В этих уравнениях функция $\chi$ определена условиями

$$
\Delta \chi=0,\left.\quad \frac{\partial \chi}{\partial n}\right|_{\partial \Omega}=x_{0}\left(\cos n x_{2}+i \cos n x_{1}\right)-\left(x_{2}+i x_{1}\right) \cos n x_{0} .
$$

Можно проверить, что функция $\chi$ удовлетворяет равенству

$$
P_{o}\left(\frac{\partial \chi}{\partial x_{2}},-\frac{\partial \chi}{\partial x_{1}}, 0\right)^{t}=(B H-G B) e, \quad e=\frac{1}{\sqrt{2}}(0,1, i)^{t},
$$

а оператор $\mathbf{F}$ есть сужение оператора $\mathbf{T}_{0}=\mathbf{S}_{0} \mathbf{T} \mathbf{S}_{0}^{-1}$ на подпространство $\mathfrak{H}_{1}$ (см. подробнее $[8,9]$ ). Тем самым при $\nu=0$ изучение оператора Соболева $\mathbf{F}$ сводится к изучению оператора $\mathbf{T}_{0}$. Нам неизвестно, как определить оператор Соболева $\mathbf{F}$ для вязкой жидкости. Трудность состоит в том, что в равенстве (16) нельзя вместо оператора $G$ взять $D$, так как вектор $B e$ не содержится в области определения оператора Стокса $R$. Действительно, если $B e \subset \mathscr{D}(R)$, то справедливо равенство $B e=[e, x]+\nabla p$, откуда получаем

$$
R B e=\Delta([e, x])+\nabla \Delta p+\nabla q=\nabla(\Delta p+q) .
$$

Здесь $p$ и $q$ - некоторые гладкие функции. Следовательно, $P_{0} R B e=R B e$ $=0$. Это противоречит условию $R \gg 0$.

Авторы благодарят В. В. Козлова, В. Б. Лидского, Е. С. Пятницкого и Ф. Л. Черноусько за полезное обсуждение работы.

\section{ЛитеРАТУРА}

1. Соболев С. Л. О движении симметричного волчка с полостью, наполненной жидкостью. ПМТФ, № 3, 20-55 (1960).

2. Румянцев B. В. Методы Ляпунова в исследовании устойчивости движения твердых тел с полостями, наполненными жидкостью. Изв. АН СССР, сер. механика и машиностроение, № 6, 119-140 (1963).

3. Черноусько Ф. Л. Движения твердого тела с полостью содержащей вязкую жидкость. ВЦ АН СССР, 1968.

4. Моисеев Н. Н., Румянцев В. В. Динамика тела с полостями, содержащими жидкость. Наука, М., 1965.

5. Копачевский Н. Д., Крейн С. Г., Нго Зуй Кан. Операторные методы в линейной гидродинамике: эволюционные спектральные задачи. Наука, М., 1989.

6. Смирнова Е. П. Устойчивость свободного вращения волчка содержащего тороидальную полость с жидкостью малой вязкости. МТТ, № 5, 20-25 (1976). 
7. Черноусько Ф. Л. Вращательные движения твердого тела с полостью, заполненной жидкостью. ПММ, 31, 416-432 (1967).

8. Юркин М. Ю. О конечномерности задачи о малых колебаниях волчка с полостью, заполненной идеальной жидкостью. Функц. анализ и его прил., $\mathbf{3 1}$, вып. 1, 51-66 (1997).

9. Юркин М. Ю. Об устойчивости вращающегося несимметричного волчка с жидким наполнением. Принята к печати в ДАН.

10. Ладыюенскал О. А. Математические вопросы динамики вязкой несжимаемой жидкости. Наука, М., 1970.

11. Азизов Т. Я., Иохвидов И. С. Основы теории линейных операторов в пространствах с индефинитной метрикой. Наука, М., 1986.

12. Гохберг И. Ц., Крейн М. Г. Введение в теорию линейных несамосопряженных операторов в гильбертовом пространстве. Наука, М., 1965.

13. Понтрягин Л. С. Эрмитовы операторы в пространстве с индефинитной метрикой. Изв. АН СССР, сер. матем., 8, № 6, 243-280 (1944).

14. Крейн М. Г., Лангер Г. К. О дефинитных подпространствах и обобщенных резольвентах эрмитовых операторов в пространствах Пћ. Функц. анализ и его прил., 5, вып. 2, 59-71 (1971); 5, вып. 3, 54-69 (1971).

15. Азизов T. Я. Диссипативные операторы в гильбертовом пространстве с индефинитной метрикой. Изв. АН СССР, сер. матем., 37, № 3, 639-662 (1973).

16. Милославский А. И. Об устойчивости некоторых классов эволюционных уравнений. Сиб. матем. ж., XXVI, № 5, 118-132 (1985).

17. Kaто T. Теория возмущений линейных операторов. Мир, М., 1972.

18. Shkalikov A. A. Operator pencils arising in elasticity and hydrodynamics: the instability index formula. In: Operator Theory: Advances and Applications, Vol. 87, Birkhäuser, 1996, pp. 258-285.

19. Шкаликов A. А. Формула индекса неустойчивости для уравнений с диссипацией. УМН, 51, вып. 5, 195-196 (1996).

20. Лидский В. Б. О суммируемости рядов по главным векторам несамосопряженных операторов. Труды МMO, 11, 3-35 (1962).

21. Shkalikov A. A. Estimates of holomorphic functions and summability theorem. Pacific J. Math., 103, No. 2, 569-582 (1982).

22. Смирнова Е. П. Стабилизация свободного вращения ассиметрического волчка с полостями, целиком заполненными жидкостью. ПММ, 38, 980-985 (1974).

Московский государственный университет им. М. В. Ломоносова
Поступило в редакцию 29 декабря 1997 г. 